\title{
Chemische und pharmakologische Untersuchungen über das Verbenin, ein galaktogog wirkendes Glykosid von Verbena officinalis $L$.
}

\author{
Von \\ Kōtoku Kuwajima. \\ (桑 島 笛 德) \\ (Aus dem Pharmakologischen Institut der Kaiserlichen Tohoku \\ Universität zu Sendai. Director : Prof. S. Yagi.)
}

Verbena officinalis L. ist ein mehrjähriges Kraut mit spindelförmigen, ästigen Wurzeln und $30-40 \mathrm{~cm}$ hohen, vierkantigen, ästigen Stengeln, die gegenständige, keilförmige oder spaltige, gesägte, rauhe Blätter tragen. Auf rutenförmigen $Z$ weigen stehen in lockeren Ähren kleine blassviolette Blüten, deren 4-5 zipfliger Kelch röhrenförmig ist. Dieses Kraut scheint in alten Zeiten sowohl im Morgen- als auch im Abendland als ein Uterus-sowie Milchmittel verwendet worden zu sein, obgleich gegenwärtig unklar ist, ob es dabei den erwünschten Erfolg gebracht hat. Bourdier1) versuchte aus diesem Kraut den wirksamen Bestandteil zu isolieren und konnte ein Glykosid, das Verbenalin, darstellen, das sich aber nach seinen Versuchen an Meerschweinchen als wirkungslos nachweisen liess. Später gelang es auch Holste (2) $^{2}$ aus derselben Droge ein Glykosid, dem er denselben Name gab, zu isolieren, das bei Meerschweinchen auf den Uterus tonussteigernd und bewegungsfördernd wirkte. Somit ist es vérständlich, dass die Droge infolge ihres Gehaltes an diesem Bestandteil bei der Behandlung der Menorrhagie, adynamischen Uterusblutungen u.s.w. gewisse Dienste leisten mag. Ob das Verbenalin auf die Milchabsonderung fördernd wirkt, ist aber ganz unklar, da er diesbezügliche Versuche nicht angestellt hat. . Deshalb bemühte ich mich, aus dem Kraut den galaktophorisch wirkenden Bestandteil chemisch rein darzustellen und konnte als denselben ein Glykosid gewinnen, das in seinen chemischen sowie 2,681 .

1) Bourdier, z. n. Abderhalden, Biochemisches Handlexikon, Berlin, 1911,

2) Hols te, Zeitschr. f. exp. Path. u. Therap. 1918, 19, 483. 
pharmakologischen Eigenschaften dem Verbenalin sehr nahe steht, aber ihm keineswegs identisch ist, so dass ihm ein neuer Namen „Verbenin" gegeben wurde. Im folgenden soll über seine chemischen und pharmakologischen Eigenschaften berichtet werden.

\section{Chemisches.}

\section{Darstellungsmethode.}

Da Versuche an melkenden Kaninchen gezeigt hatten, dass subkutane Einverleibung eines wässerigen Auszuges von Verbena officinalis L. die Milchabsonderung deutlich vermehrte, wurde eine gewisse Menge des getrockneten pulverisierten Krautes zuerst in einem Soxhlet schen Apparat mit Petroleumäther, Äther und absolutem Alkohol nacheinander und dann in einem Kolben mit heissem Wasser extrahiert, um festzustellen, in welches Lösungsmittel der wirksame Bestandteil übergeht. Jede Fraktion wurde nach der richtigen Behandlung melkenden Tieren subkutan dargereicht und ihr Einfluss auf die Milchabsonderung beobachtet. Dabei wurde gefunden, dass die ätherische Fraktion eine deutliche und die alkoholische eine leichte galaktogoge Wirkung zeigte, während sich bei der petroleumätherischen und wässerigen Fraktion keine solche nachweisen liessen. Deshalb wurde das Kraut bei der. Isolierung des wirksamen Bestandteils zuerst mit Äther behandelt.

Das pulverisierte Kraut wurde in einem Soxhlet schen Apparat mit Äther so lange extrahiert, bis sein bitterer Geschmack zum grossen Teil verloren gegangen war. Der Inhalt des Kolbens wurde, nachdem der darin gebliebene Äther abgekühlt und abgegossen worden war, mit kaltem Äther wiederholt ausgewaschen, bis er sich nicht mehr färbte. Der zurückgebliebene, grauweisse Niederschlag wurde in Wasser gelöst, die Lösung nach Filtration auf einem Wasserbad eingedampft und der Rückstand in absolutem Alkohol aufgenommen. Beim Verdunsten der alkoholischen Lösung schied sich eine weiss kristallinische Masse aus, die durch mehrmalige Umkristallisationen aus absolutem Alkohol gereinigt wurde. Die dadurch erhaltene Masse bestand zum grössten Teil aus tafelförmigen, und zum kleinsten Teil aus nadelförmigen Kristallen, die beiden konnten aber durch fraktionierte Kristallisation aus Alkohol voneinander getrennt werden. Bei der Darreichung an melkende Tiere verursachte die tafelförmige Substanz eine ziemlich starke, die nadelförmige hingegen eine ganz schwache Vermehrung der Milchsekretion. Die galaktogoge Wirkung des Krautes 
ist also hauptsächlich der Anwesenheit der tafelförmigen Substanz zuzuschreiben, obgleich die nadelförmige einigermassen daran beteiligt sein kann.

\section{Eigenschaften.}

Die eine der obengenannten Substanzen kristallisiert in farblosen länglich viereckigen Täfelchen und schmilzt bei $179^{\circ} \mathrm{C}$, während die andere farblose nadelförmige Prismen bildet und einen Schmelzpunkt von $177^{\circ} \mathrm{C}$ besitzt. Die beiden schmecken sehr bitter und lösen sich sehr leicht in Wasser, leicht in absolutem Alkohol, schwer in Äther und sehr schwer in Chloroform. Thre wässerigen Lösungen reagieren neutral. Die beiden Substanzen reduzieren die Fehling sche Lösung und ammoniakalische Silbernitratlösung in der Kälte nicht, wohl aber in der Wärme, besonders schnell nach Behandlung int Salzsäure. Bei längerem Kochen dieser Substanzen mit verdünnter Schwefelsäure scheidet sich ein hellgelber, amorpher Niederschlag aus. Kocht man diese Substanzen mit Eisessig und Phenylhydrazin, so entsteht allmählich eine bräunlichrote Ausscheidung.

Aus dem gesagten erhellt, dass die beiden Substanzen Glykoside sind, deren chemische Eigenschaften mit denen des Verbenalin von Bourdier und von Holste sehr gut übereinstimmen. Von den zwei Glykosiden scheint das nadelförmige dem Verbenalin identisch zu sein, das letztere soll nach den genannten Autoren in Nadeln kristallisieren und nach Bourdier bei $181^{\circ} \mathrm{C}$ und nach Hols te bei $178^{\circ} \mathrm{C}$ schmelzen, obgleich eine pharmakologische Identitätsbestimmung der beiden Substanzen wegen des Mangels an Material nicht ausgeführt worden ist. Dagegen ist das tafelförmige Glykosid, das für die galaktogoge Wirkung des Krautes verantwortlich ist, vom Verbenalin nicht nur in der Kristallform, sondern, wie später berichtet wird, auch in den pharmakologischen Wirkungen verschieden, wenn sein Schmelzpunkt auch dem des Verbenalin sehr nahe steht. Infolgedessen muss man annehmen, dass das tafelförmige Glokosid ein neues ist, was mich veranlasste, ihm den neuen Namen „Verbenin" zu geben. Woher es kommt, dass Verf. aus dem Kraut neben dem bekannten Verbenalin noch das Verbenin, das von den früheren Autoren nicht bemerkt wurde, darstellen komnte, ist vorläufig unklar. Es ist möglich, dass die $\mathrm{Zu}$ sammensetzung der Droge je nach ihrer Heimat verschieden ist. Doch könnte es vielmehr darauf beruhen, dass Verf. bei der Darstellung der wirksamen Bestandteile eine ganz andere Methode verwendete als die 
der früheren Autoren, woüber bei anderer Gelegenheit noch näher berichtet werden soll.

\section{Pharmakologisches.}

1. Versuche an Fröschen.

1) Allgemeines Vergiftungsbild. Versuche wurden an Fröschen (Rana japonica) in der Monaten Juli und August angestellt, indem das Verbenin in den Bauchlymphsack einverleibt wurde. Bei Dosen von 2-5 mg pro $10 \mathrm{~g}$ Körpergewicht, die eine nennenswerte Veränderung der willkürlichen, Reflex- und Atembewegungen hervorzurufen nicht imstande waren, bemerkte man zuerst Anschwellen der Schleimdruisen der Haut und dann Feuchterwerden derselben. Die Feuchtigkeit wurde manchmal so auffallend, dass die ganze Hautoberfläche mit Schaum bedeckt war. Diese Erscheinung hielt 30-60 Minuten an. Bei Dosen von 10-50 $\mathrm{mg}$ erfolgte neben einer unbedeutenden Trägheit der willkürlichen Bewegungen ebenfalls eine Vermehrung der Schleimabsonderung, die aber schneller einsetzte, 10-30 Minuten anhielt und dann einer Verminderung derselben Platz machte. Bei Dosen uber $100 \mathrm{mg}$ war die Vermehrung der Hautsekretion nicht deutlich und schlug etwa 10 Minuten danach ins gegenteilige Stadium um, das lange Zeit andauerte. Hierbei wurden die willkürlichen sowie die Reflexbewegungen immer träger und die Atembewegungen immer langsamer und schwächer, so dass sie bei einer recht grossen Dosis wie $300 \mathrm{mg}$ schliesslich völlig aufhörten. In dieser Zeit schlug das Herz noch regelmässig und kräftig fort und antwortete der Gastroknemius auf faradische Reizung des betreffenden Ischiadikus prompt mit kräftigen Kontraktionen. Erst später erfuhren die Herzschläge eine aurikuloventrikuläre Dissoziation und hörten mehrere Stunden nach der Darreichung auf.

Aus diesen Versuchen ist es verständlich, dass das Verbenin bei Fröschen in kleinen Dosen auf die Schleimdrüsen der Haut sekretionsfördernd, in grossen auf das Zentralnervensystem lähmend und in recht grossen auf das Herz schädigend wirkt. Nach Holste soll das Verbenalin an Fröschen einerseits eine Vermehrung der Schleimabsonderung wie das Verbenin, andererseits zum Tetanus entwickelte, klonische Krämpfe bewirken, die beim letzteren aber niemals erfolgen. Dies ist ein Grund dafür, dass Verf. das Verbenin als ein neues Glykosid betrachtet. Jedenfalls kann man sagen, dass die Hauptwirkung des Verbenins auf Frösche in der Vermehrung der Schleimabsonderung 
liegt, da die sonstigen Erscheinungen erst in sehr grossen Dosen zum Vorschein kommen. Deshalb wurde im folgenden der Mechanismus, mit dem das Verbenin die Hautsekretion fördert, untersucht.

2) Wirkung auf die Hautsekretion. Da das Verbenin wie das Verbenalin auf die Schleimdrüsen wirkt, indem es in kleinen Dosen ihre Sekretion vermehrt, aber in grossen dagegen dieselbe vermindert, so dürfte der Wirkungsmechanismus des ersteren vielleicht dem des letzteren identisch sein. Der Wirkungsmechanismus des Verbenalins ist aber ganz unklar, da Holste keine diesbezüglichen Versuche vorgenommen hat.

Wurden $5 \mathrm{mg}$ Verbenalin pro $10 \mathrm{~g}$ Körpergewicht in den Bauchlymphsack der Frösche, deren Gehirn 1 Stunde vorher exstirpiert worden war, injiziert, so vermehrte sich die Schleimabsonderung der Haut ganz wie bei den unversehrten Tieren. Injektion des Glykosides von der genannten Dosis in denselben Lymphsack der Tiere, bei denen der Ischiadikus an einem Oberschenkel durchschnitten worden und die Haut des betreffenden Beingebietes deutlich trockener als die des entsprechenden Gebietes des anderen Beins geworden war, bewirkte auf beiden Seiten eine Vermehrung der Schleimabsonderung, wenn auch bei ersterem der Grad der Vermehrung etwas schwächer und die Dauer einigermassen kürzer als bei letzterem war. Die Ergebnisse dieser Versuche lassen sich dadurch erklären, dass die durch das Verbenin herbeigeführte Vermehrung der Schleimabsonderung die Folge der Erregung nicht des sekretorischen Zentrum, sondern irgendeines peripherischen Apparates ist.

Bei Fröschen, deren Hautoberfläche durch Darreichung von $1 \mathrm{mg}$ Atropin pro $10 \mathrm{~g}$ Körpergewicht in den Oberschenkellymphsack trockener geworden war und bei denen Injektion von $1 \mathrm{mg}$ Pilokarpin in den Bauchlymphsack nicht imstande war, eine Vermehrung der Hautsekretion hervorzurufen, hatte die Injektion von $5 \mathrm{mg}$ Verbenin eine deutliche Vermehrung derselben zur Folge. Wenn Fröschen $20 \mathrm{mg}$ Agaricinsäure in den Oberschenkellymphsack gegeben wurde, so wurde die Hautoberfläche allmählich trocken und sah etwa 1 Stunde nach der Darreichung ganz dürr aus. In diesem Stadium verursachte weder Adrenalin noch Verbenin eine Vermehrung der Schleimabsonderung. Die Stelle, auf die das Verbenin sekretionsfördernd wirkt, scheint also entweder in den sympathischen Nervenenden oder in den Drüsenzellen zu liegen.

Wie erwähnt, wurde die äussere Haut der Frösche nach Darreichung des Verbenins in grossen Dosen, wie $100 \mathrm{mg}$ pro kg Körperge- 
wicht, ohne feuchter zu werden, von Anfang an trockener. Etwa 1 Stunde nach der Darreichung war die subkutane Injektion von $0,1 \mathrm{mg}$ Adrenalin, die sonst eine bedeutende Vermehrung der Hautsekretion herbeizuführen vermochte, nicht mehr imstande, diese hervorzurufen, während die von $1 \mathrm{mg}$ Pilokarpin eine profuse Hautsekretion zur Folge hatte, wenn sie auch etwas schwächer ausfiel als die bei den nicht mit Verbenin vorbehandelten Tieren. Es ist also sicher, dass das Verbenin in grossen Dosen die sympathischen Nervenenden der Schleimdrüsen lähmt und dadurch eine Sekretionsverminderung verursacht. Höchst wahrscheinlich liegt auch die Stelle, auf die das Glykosid in kleinen Dosen sekretionsfördernd wirkt, nicht in den Drüsenzellen, sondern in denselben Nervenenden. $\mathrm{Ob}$ diese Vermutung zutreffend ist, soll durch weitere Untersuchungen an anderen Organen induktiv festgestellt werden.

3) Wirkung auf das Herz. Bei der Registrierung des blossgelegten Herzens kurarisierter Frösche bemerkte man, dass die Herzschläge einige Minuten nach Injektion von $5 \mathrm{mg}$ Verbenin pro $10 \mathrm{~g}$ Gewicht in den Schenkellymphsack sowohl in der Frequenz als auch im Umfang, wenn auch nicht erheblich, so doch in allen Fällen zunahmen. Diese Veränderungen kehrten etwa 10 Minuten danach zurück. Bei einer Dosis von $30 \mathrm{mg}$ erfolgte ebenfalls eine leichte Amplitudenvergrösserung mit einer geringen Frequenzzunahme, die hierbei aber bald ins Gegenteil umschlug. Dieselben Veränderungen wurden bei Versuchen am isolierten Froschherzen mit 0,005 oder 0,01\%iger Verbeninlösung beobachtet. Wenn das isolierte Herz aber vorher mit einer 0,001\%igen Ergotoxinlösung vorbehandelt worden war, waren die Verbeninlösungen nicht mehr imstande, die genannten Veränderungen der Herzschläge herbeizuführen. Wenn die Schläge eines isolierten Herzens durch Ein wirkung einer 0,01\%igen Verbeninlösung verlangsamt worden waren, verursachte eine $0,0001 \%$ ige Adrenalinlösung keine Frequenzzunahme und Amplitudenvergrösserung, die bei den nicht mit dem Verbenin vorbehandelten Herzen durch dieselbe Lösung hervorgebracht zu werden pflegten. Aus diesen Versuchen erhellt, dass das Verbenin auf die sympathischen Nervenenden des Froschherzens in kleinen Dosen erregend, in grossen dagegen lähmend wirkt. Dieses Ergebnis scheint die Vermutung mittelbar zu bestätigen, dass das Glykosid bei den Schleimdrüsen der Haut auf die gleichnamigen Nervenenden, die durch grosse Dosen desselben gelähmt werden, in kleinen erregend einwirkt, um die Schleimsekretion zu vermehren. 
4) Wirkung auf die isolierten Schenkelgefässe. Bei Durchströmungsversuchen an isolierten Schenkelgefässen von Fröschen erfolgte nach der Injektion von $0,1 \mathrm{ccm}$ einer $0,1 \%$ igen Verbeninlösung in den zuführenden Gummischlauch eine leichte Verminderung der Ausflussflüssigkeit, die 5-6 Minuten lang anhielt. Die Verminderung wurde mit einer Konzentrationssteigerung der zu prüfenden Lösung deutlich, ging aber, wenn die Konzentration 0,4\% erreichte, nach einigen Minuten in eine leichte Vermehrung uber, die etwa 10 Minuten lang bestehen blieb. Bei Verwendung einer 1\%igen Lösung trat ohne eine vorangehende Verminderung von Anfang an eine leichte Vermehrung derselben ein, der aber früher oder später eine geringe, jedoch lang andauernde Verminderung folgte.

In Durchströmungsversuchen, bei denen 0,1 ccm einer $0,02 \%$ igen Ergotoxinlösung in den zuführende Gummischlauch wiederholt eingespritzt worden war, bewirkte die Injection der $0,1 \%$ igen Verbeninlösung keine Verminderung der Ausflussfluigkeit, wohl aber eine leichte Vermehrung, während die einer 1\%igen Lösung ebenfalls eine Vermehrung und darauffolgende Verminderung zur Folge hatte. Bei Schenkelpräparaten, deren Ausflussflüssigkeit durch wiederholte Injektionen von 1\%igen Verbenalinlösungen deutlich vermindert worden war, führte $0,1 \mathrm{ccm}$ einer Adrenalinlösung von 1:100 000 keine Verminderung, ja sogar manchmal eine leichte Vermehrung der Ausflussfluissigkeit herbei.

Diese Versuche beweisen, dass das Verbenin bei den Gefässen in niedrigen Konzentrationen auf die fördernden sympathischen Nervenenden erregend, in hohen dagegen auf dieselben lähmend und gleichzeitig damit auf die Muskeln erregend wirkt. Auch dies scheint die Vermutung zu unterstützen, dass das Glykosid auf die sympathischen Nervenenden der Schleimdrüsen in kleinen Dosen erregend und in grossen lähmend einwirkt.

5) Wirkung auf die Pupille. Die Pupille eines herausgeschnittenen, in Ringerlösung getauchten Augapfels zeigte nach Zusatz von Verbenin in die Nährflüssigkeit in einer Konzentration von 0,01\% eine geringe Erweiterung, die aber selbst bei Steigerung der Konzentration auf $1 \%$ nicht erheblich zunahm. Die Pupille eines isolierten Augapfels, der in der Glykosidlösung von der letztgenannten Konzentration längere Zeit gelegen hatte, zeigte nach Zusatz von Adrenali im Verhältnis 1:000 000, der sonst eine deutliche Erweiterung hervorzurufen vermochte, keine solche, während sie auf Żusatz von Pilokarpin im Verhältnis 1:10000 mit einer starken Verengerung 
antwortete, ganz wie bei der nicht vorbehandelten Pupille. Bei einem Augapfel, dessen Pupille durch Einwirkung einer 0,1\%igen Atropinlösung stark erweitert worden war, führte eine $0,1 \%$ ige Verbeninlösung eine weitere, wenn auch sehr schwache, jedoch unverkennbare Erweiterung herbei.

Das Verbenin wirkt also auch auf die Pupille, indem es die sympathischen Nervenenden zuerst erregt und dann lähmt. Es ist deshalb sehr wahrscheinlich, dass es auch bei den Schleimdrüsen deren sympathische Nervenenden zuerst erregt und dann lähmt.

\section{Versuche an Mäusen.}

Die kleinste subkutane Dosis des Verbenins, die an Mäusen eine bemerkbare Veränderung des Allgemeinzustandes hervorzubringen vermochte, lag bei $30 \mathrm{mg}$ pro $10 \mathrm{~g}$ Körpergewicht, bei denen sich die Tiere etwa 30 Minuten lang ruhig verhielten, ohne jedoch sonstige Vergiftungserscheinungen zu zeigen. Diese Vergiftungserscheinungen nahmen mit der Vermehrung der Dosis immer zu, so dass bei $100 \mathrm{mg}$ die Tiere auf dem Bauche lagen, ohne spontane Bewegungen auszuführen, obgleich sie bei mechanischer Reizung vergeblich vom Reiz wegzurücken versuchten. Dabei waren die A tembewegungen sehr langsam, aber regelmässig und ziemlich stark. In einigen Fällen wurde auch ein deutlicher Speichelfluss beobachtet, während sich in anderen kein solcher nachweisen liess. Von diesen Vergiftungserscheinungen erholten sich die Tiere 3-4 Stunden nach der Darreichung anscheinend vollständig. Bei $200 \mathrm{mg}$, die ähnliche Vergiftungserscheinungen verursachten, wurden die Atembewegungen anfänglich langsam, dann aber frequent und angestrengt, später wiederum langsam und flach, und kamen schliesslich unter schwachen Konvulsionen zum Stillstand. Dabei war Ausführung künstlicher Atmung imstande, das Herz lange Zeit danach regelmässig und ziemlich kräftig fortschlagen zu lassen.

Das Verbenin bewirkt also an Mäusen wie an Fröschen eine motorische Lähmung, die vielleicht von zentraler Natur ist. Die kurz vor dem Tode eintretenden Konvulsionen sind nicht als die Folge der Erregung des motorischen Apparates durch das Glykosid selbst, wohl aber durch die Erstickung zu betrachten. Das Glykosid scheint überdies noch einen Speichelfluss herbeizuführen. Die erste Todesursache bei der Vergiftung liegt sicherlich im Respirationsstillstand. 


\section{Versuche an Kaninchen.}

Bei Kaninchen liess sich nach intravenöser Darreichung von $1 \mathrm{~g}$ Verbenin pro kg Körpergewicht gar keine Vergiftungserscheinung bemerken. Dies hängt vielleicht nur davon ab, dass die verwendete Menge zu klein ist, denn das Glykosid soll, wenn aus dem Ergebnis der Versuche an Mäusen zu schliessen erlaubt ist, in einer Dosis von $3 \mathrm{~g}$ pro $\mathrm{kg}$ Gewicht eine bemerkbare und erst in einer grossen wie $20 \mathrm{~g}$ tödliche Vergiftungserscheinungen herbeiführen. Wegen Mangels am Material konnten aber weitere Untersuchungen über die allgemeinen Vergiftungserscheinungen nicht angestellt werden. Wie oben erörtert, soll das Verbenalin nach $\mathrm{Holste}$ auf den isolierten Uterus von Meerschweinchen eine starke erregende Wirkung ausüben. So ist es wahrscheinlich, dass dem Verbenin, dessen chemische Eigenschaften denen des Verbenalins sehrnahe verwandt sind, ebenfalls eine uteruserregende Wirkung zuzuschreiben ist. Dies kann aber noch nicht sicher sein, wenn man daran denkt, dass jenes Glykosid im Unterschied von diesem keine krampferzeugende Wirkung hat. Deshalb beschäftigte Verf. sich zunächst mit dieser Frage.

1) Wirkung auf den Uterus. Weibliche Kaninchen von etwa 2,5 kg Körpergewicht wurden unter Äthernarkose in der Rückenlage befestigt und der Uterus nach der Methode von To y o $\mathrm{m} \mathrm{a}^{3)}$ zur Registrierung des Darmzustandes in situ mittels eines Fadens mit einem Schreibhebel verbunden, um seinen Zustand wiedergeben zu lassen. Gleichzeitig damit wurden in einigen Fällen auch der Karotisdruck mittels eines Hg-Manometers oder die Darmbewegungen nach der oben bezeichneten Methode registriert. Die zu prüfende Lösung wurde immer in die Ohrvene eingespritzt.

Bei nicht trächtigen Tieren bewirkte das Verbenin in Dosen unter $10 \mathrm{mg}$ pro kg Gewicht keine Veränderung des Uterus weder im Tonus noch in den rhythmischen Bewegungen. Erst in einer Dosis von 20 $\mathrm{mg}$ zeigten die Uterusbewegungen in den meisten Fällen eine leichte, aber langanhaltende Amplitudenvergrösserung, die eine Verstärkung sowohl der Kontraktion als auch der Erschlaffung betraf, während in seltenen Füllen keine solche zur Erscheinung kam. Bei $50 \mathrm{mg}$ war die Kontraktion noch etwas stärker, während die Erschlaffung dabei vielmehr eine leichte Beschränkung erfuhr. Diese war bei $100 \mathrm{mg}$ deutlich, so dass dann eine bedeutende Tonuszunahme mit einer starken

3) Toy o ma, Tohoku journ. of exp. med. 1933, 22, 196. 
Amplitudenverkleinerung erfolgte, die aber den Uterus niemals zum Tetanus führte. In den angegebenen Dosen führte das Glykosid weder im Blutdruck noch in der Dünndarmbewegungen nennenswerte Veränderungen herbei.

Das Verbenin hat also, wie das Verbenalin, eine uteruserregende Wirkung, die aber im Vergleich mit den bekannten Uterustonika keineswegs stark ist, so dass es gegenwärtig als solches zum therapeutischen Zwecke kaum in Betracht kommen kann. Da das Verbenalin nach Holste eine starke, dem Hypophysis-Optonen und Tenosin ebenbürtige uteruserregende Wirkung besitzt, so scheint das Verbenin in der Uteruswirkung weit hinter dem ersteren zurückzustehen. Jedenfalls ist es sicher, dass das Verbenin wie das Verbenalin an der Uteruswirkung der Droge beteiligt ist, obgleich es vorläufig noch unbekannt ist, welches von den beiden dabei die Hauptrolle spielt. Um nun den Mechanismus der Uteruswirkung des Verbenins festzustellen, wurden Versuche am herausgeschnittenenUterus erwachsener, aber nicht trächtiger Kaninchen vorgenommen.

Ein Uterusstück wurde in einem erwärmten, mit Sauerstoff durchleiteten Ringerbad von $100 \mathrm{ccm}$ suspendiert, um seinen Zustand zu registrieren. Die zu prüfende Verbeninlösung wurde zu geeigneter Zeit dem Bad zugesetzt. Das Glykosid verursachte in einer Dosis von 10 mg eine Vergrösserung der Kontraktion und Erschlaffung, in einer solchen von $50 \mathrm{mg}$ eine Verstärkung der ersteren mit einer Beschränkung der letzteren und in einer solchen von $100 \mathrm{mg}$ eine Tonussteigerung mit einer Amplitudenverkleinerung, ganz wie beim Uterus in situ. Bei grösseren Dosen, wie $200 \mathrm{mg}$, ging der Tonussteigerung eine flüchtige leichte Tonusherabsetzung voran. Dieselben Zustandsänderungen wurden bei einem Uterusstück, das vorher mit $1 \mathrm{mg}$ Atropin vergiftet worden war, durch das Gift von den obenbezeichneten Dosen hervorgebracht. Bei einem Uterusstück, das vorher mit $1 \mathrm{mg}$ Ergotoxin behandelt worden war, verursachte das Glykosid in Dosen von 10-50 mg gar oder fast keine Zustandsänderung und in Dosen über 100 mg eine allmählich eintretende leichte Tonussteigerung, die aber durch $1 \mathrm{mg}$ Atropin nicht beseitigt werden konnte. Wenn ein Uterusstück einige Minuten vorher mit $200 \mathrm{mg}$ Verbenin vergiftet worden war, so verursachte $0,1 \mathrm{mg}$ Adrenalin keine weitere Zunahme des Tonus, sondern dagegen eine, wenn auch nicht sehr starke, so doch deutliche Herabsetzung desselben.

Diese Versuche zeigen, dass das Verbenin beim Kaninchenuterus in kleinen Dosen die fördernden sympathischen Nervenenden erregt, 
in grossen dieselben lähmt und gleichzeitig damit die kontraktilen Elemente erregt, so dass die durch das Glykosid herbeigeführte Zustandsänderung je nach den Dosen verschieden ist. Es wirkt aber nicht auf die hemmenden sympathischen Nervenenden, worauf beruhen könnte, dass das Glykosid trotz seiner unverkennbaren Uteruswirkung auf den Darm, dessen sympathische Nerven fast ausschliesslich aus hemmenden Fasern' bestehen, keine nennenswerte Zustandsünderung herbeiführt.

2) Wirkung auf die Speichelsekretion. Aus der Beobachtung, dass das Verbenin bei Kaninchen keinen Speichelfluss herbeiführte, kann man doch noch nicht ohne weiteres schliessen, dass es keine speichelfördernde Wirkung hat, da sich bei Mäusen manchmal eine solche beobachten liess. Um diese Frage zu lösen, wurden Versuche an mit Äther narkotisierten Kaninchen angestellt, bei denen in den Ausführungsgang der Submaxillardrüse einer Seite nach Blosslegung eine Glaskanüle, die mit einem fein graduierten Glasrohr von etwa $0,5 \mathrm{~mm}$ Durchmesser in Verbindung stand, hineingesteckt wurde, um die Geschwindigkeit der Speichelabsonderung genau beobachten zu können, Die intravenöse Darreichung von Verbenin in Dosen über $10 \mathrm{mg}$ pro kg Körpergewicht bewirkte eine kurz dauernde Vermehrung der Speichelsekretion, die mit Steigerung der Dosis auffallender wurde, so dass sich die Speichelmenge pro Minute bei einer Dosis von $50 \mathrm{mg}$ auf das 10-20 fache der anfänglichen vermehrte. Steigerte man die Menge aber darüber, so wurde die Vermehrung mit der Menge immer weniger auffallender, so dass bei $100 \mathrm{mg}$ fast keine Vermehrung, ja sogar eine deutliche Verminderung erfolgte. In diesem Zustand war die intravenöse Injektion von $0,02 \mathrm{mg}$ Adrenalin, das sonst eine deutlicheVermehrung der Speichelsekretion herbeizuführen vermochte, nicht mehr imstande, eine solche hervorzurufen, während die von 0,2 $\mathrm{mg}$ Pilokarpin wie bei den normalen Tieren eine starke Speichelvermehrung zur Folge hatte. Bei Tieren, denen vorher $10 \mathrm{mg}$ Atropin subkutan gegeben worden war, führte das Glykosid in Dosen von 10-50 mg ebenfalls eine Speichelvermehrung herbei, wie bei nicht atropinisierten Tieren.

Aus diesen Versuchen geht hervor, dass das Verbenin in kleinen Dosen durch Erregung der sympathischen Nerven die Speichelsekretion vermehrt, in grossen dagegen durch Lähmung derselben Nerven die Sekretion vermindert. Sein Angriffspunkt soll nach den bisherigen Untersuchungen auch bei den Speicheldrüsen in den Nervenenden liegen. Seine speichelvermehrende Wirkung ist aber nicht sehr stark und 
zwar kurz anhaltend, so dass kein Speichelfluss erfolgen kann. Dass das Glykosid trotz seiner sympathikuserregenden Wirkung keine nennenswerte Veränderung des Blutdruckes verursacht, könnte vielleicht davon abhängen, dass die Wirkung sehr flüchtigt ist.

3) Wirkung auf die Milchsekretion. In der Einleitung wurde schon gesagt, dass das Verbenin bei weiblichen melkenden Kaninchen die Milchsekretion steigert, woüber hier noch näher berichtet werden soll. Weibliche Kaninchen, die täglich gleichmässig gefüttert wurden, wurden 2 Wochen nach der Entbindung von den Jungen getrennt und danach alle Tage von $8 \mathrm{Uhr}$ vormittags bis $4 \mathrm{Uhr}$ nachmittags stündlich einmal künstlich gemolken, indem die einzelnen Milchdrüsen mit warmen Fingern sanft gepresst und die heraussickernde Milch mit einer Pipette aufgesaugt wurde. Die tägliche Menge der auf diese Weise erhaltenen Milch war je nach den einzelnen Tieren ziemlich verschieden, indem sie zwischen 26 und $61 \mathrm{ccm}$ schwankte. Bei ein und demselben Tier aber blieb die Schwankung der täglichen sowie der stündlichen Milchmenge innerhalb 10\% der durchschnittlichen.

Injizierte man solchen Tieren gleich nach der dritten Melkung 10 $\mathrm{mg}$ Verbenin pro $\mathrm{kg}$ Körpergewicht intravenös, so fand man ohne Ausnahme eine Vermehrung der stündlichen Milchmenge, die schon in der ersten Stunde nach der Darreichung auffallend war und in der zweiten Stunde ihr Maximum erreichte, wobei sie $40-50 \%$ der anfänglichen Durchschnittsmenge betrug, und dann allmählich zurückging, so dass sie selbst 6 Stunden nach der Darreichung noch $20-30 \%$ zeigte. Sie dauerte aber keine 24 Stunden lang an. Die tägliche Milchmenge zeigte im Durchschnitt von 5 Fällen eine Vermehrung von etwa 24\%. Weitere Daireichung derselben Menge täglich einmal 9 Tage lang bewirkte jedesmal eine Vermehrung, die in der Intensität und Dauer der der erstmaligen Darreichung beinahe gleich war. Bei $50 \mathrm{mg}$ erreichte die Vermehrung der stündlichen Milchmenge in der ersten Stunde nach der Darreichung ihr Maximum, wobei sie 55-114\% der anfänglichen durchschnittlichen Menge betrug, dann hielt sie ungefähr in demselben Zustand wenigstens 6 Stunden an und liess sich selbst 24 Stunden nach der Darreichung noch deutlich erkennen, wie Tabelle 1 zeigt. Dabei machte die Vermehrung der täglichen Milchmenge im Durchschnitt von 5 Versuchen $71 \%$ der ursprünglichen aus. Subkutane sowie stomachale Darreichung des Verbenins verursachte eine ähnliche Veränderung der Milchsekretion, die*aber langsamer verlief als bei der intravenösen Darreichung. Bei Muttertieren, deren Milchsekretion auf- 
gehört hatte, war das Glykosid in den oben angegebenen Dosen nicht mehr imstande, die Sekretion wieder beginnen zu lassen. In den sämtlichen Versuchen liess sich keine nennenswerte Vexänderung seitens der allgemeinen Erscheinungen nachweisen.

\section{Tabelle 1.}

Stündliche Milchmenge von 5 Kaninehen von 2,5-2,9 kg Körpergewicht vor und nach der Verbenindarreichung. 5 Minuten nach 10

Uhr vormittags intravenöse Injektion von $50 \mathrm{mg}$ Verbenin pro kg Körpergẽwicht.

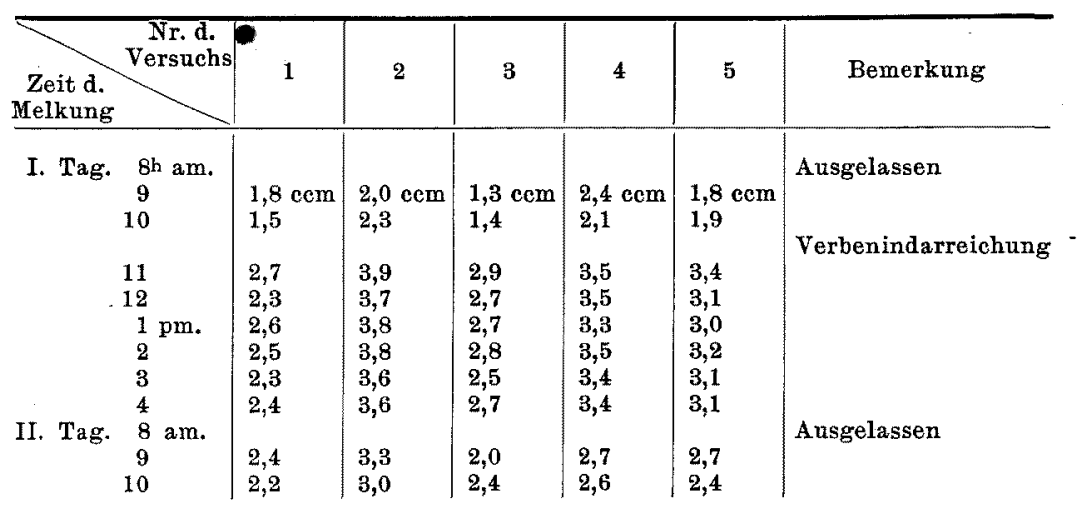

Aus diesen Versuchen ergibt sich, dass das Verbenin eine galaktogoge Wirkung hat, wie der Hypophysen- und der Plazentalextrakt. Das erstere unterscheidet sich aber von den letzteren dadurch, dass bei diesen nach Angaben von Hammond, ${ }^{4}$ ) von Hill und Simpson ${ }^{5}$ ? und von Lederer und Pribra $\mathrm{m}^{6}$ die Vermehrung der Milchsekretion kurz vorübergehend ist und von einer Verminderung derselben gefolgt wird, so dass die tägliche Milchmenge fast keine Veränderung erfährt, während bei jenem nach der Vermehrung keine Verminderung folgt, so dass sich die tägliche Milchmenge immer vermehrt, wie oben erwähnt. Obgleich die Milchsekretion von zahlreichen nervösen Einflüssen beherrscht wird, so ist doch allbekannt, dass die Innervation der Milchdrüsen von der anderer Drüsen durchaus verschieden ist, denn die autonomen Nervengifte üben auf die Milchsekretion keinen Einfluss aus. Wenn das Glykosid auch bei den Speicheldrüsen durch Erregung

4) H a m mond, Quart. J. Exp. Physiol., 1913, 6, 311.

5) Hillu. Simpson, Proc. Soc. Exp. Biol. Med., 1914, 11, 82.

6) Lederer u. Pribram, Arch. f. d. ges. Physiol., 1910, 134, 531. 
der sympathischen Nervenenden ihre Sekretion fördert, so kann man doch noch nicht daraus schliessen, dass die durch das Glykosid herbeigeführte Vermehrung der Milchsekretion auf dieselbe Weise zustande kommt. Um so weniger, wenn man bedenkt, dass die genannte Milchvermehrung auch bei grossen Dosen von Verbenin, die die sympathischen Nervenenden der Speicheldrüsen lähmen, erfolgt und im Unterschied zur Speichelvermehrung keine Verminderung folgt. Infolgedessen muss das Glykosid entweder direkt oder mittelbar durch Finwirkung auf irgendein anderes Organ die Drüsenzellen erregen, um ihre Sekretion zu steigern. Welcher von den beiden Mechanismen sich nun diesem Falle anpasst, bleibt vorläufig unentschieden. Jedenfalls ist es wünschenswert, die Beschaffenheit der nach der Verbenindarreichung sezernierten Milch genau zu untersuchen, was aber bei Kaninchenversuchen wegen der geringen Milchmenge schwierig erscheint. Deshalb wurden hier nur einige einfache Beobachtungen an der Beschaffenheit der Milch von Tieren angestellt, denen $50 \mathrm{mg}$ Verbenin pro kg Gewicht intravenös gegeben wurden.

Die Milch stellte nach der Einverleibung wie vor derselben eine undurchsichtige weisse Flỉssigkeit dar, die auf Lackmus amphoter reagierte. Auch bei nephelometrischer Untersuchung liss sich kein sicherer Unterschield in der Trübung der Milch vor und nach der Darreichung nachweisen. Gewichtsbestimmung der Trockensubstanz zeigte, dass sie bei der Milch, die 2 Stunden nach der Darreichung entnommen wurde, immer kleiner war als bei der vor derselben. Dieser Unterschied überschritt aber niemals $2 \%$ der Trockensubstanz der vor der Darreichung sezernierten Milch, während die Vermehrung der stündlichen Milchmenge nach der Darreichung 63-99\% der vor der Darreichung betrug, wie Tabelle 2 zeigt.

Aus diesen Versuchen erhellt, dass die Milch durch das Verbenin an ihren festen Stoffen eine Verminderung erfährt, die aber so klein ist, dass sie sich mit der Vermehrung der entspre-

\begin{tabular}{|c|c|c|c|}
\hline $\begin{array}{r}\text { Trocken } \\
\text { von } 2 \\
\text { nacl }\end{array}$ & $\begin{array}{l}\text { bstanz der } \\
-2,9 \mathrm{~kg} \text { Kör } \\
\text { der intraven } \\
\text { on } 50 \mathrm{mg} \text { Ve } \\
\text { Körperg }\end{array}$ & $\begin{array}{l}\text { Milch von } 5 \\
\text { pergewicht } \\
\text { ösen Darrei } \\
\text { rbenin pro } \\
\text { gewicht. }\end{array}$ & $\begin{array}{l}\text { Kaninchen } \\
\text { ror und } \\
\text { chung } \\
\text { cg }\end{array}$ \\
\hline \multirow{2}{*}{$\begin{array}{l}\text { Nr. d. } \\
\text { Versuchs }\end{array}$} & \multirow{2}{*}{$\begin{array}{c}\text { Vermehrung } \\
\text { d. } \\
\text { stündlichen } \\
\text { Milchmenge } \\
\text { in } \%\end{array}$} & \multicolumn{2}{|c|}{$\begin{array}{c}\text { Trockensubstanz in } 1 \mathrm{ccm} \\
\text { Milch in } \mathrm{g}\end{array}$} \\
\hline & & $\begin{array}{c}\text { vor d. } \\
\text { Darreichung }\end{array}$ & $\begin{array}{c}\text { nach d. } \\
\text { Darreichung }\end{array}$ \\
\hline 1 & 63 & 0,31293 & 0,30862 \\
\hline 2 & 99 & 0,33876 & 0,33271 \\
\hline 3 & 70 & 0,32530 & 0,32464 \\
\hline 4 & 83 & 0,32005 & 0,31873 \\
\hline 5 & 81 & 0,31879 & 0,30951 \\
\hline
\end{tabular}


chenden stündlichen Milchmenge gar nicht vergleichen lässt. Infolgedessen kann man annehmen, dass das Glykosid die Sekretion der Milch ungefähr in ihrer ursprünglichen Zusammensetzung steigert.

Da bekannt ist, dass verschiedene einverleibte körperfremdeStoffe in der Milch sezerniert werden, so ist es möglich, dass die Milch nach der Darreichung des Verbenins dasselbe enthält. Um diese Frage zu lösen, wurde ein Stück Filterpapier mit etwa $10 \mathrm{ccm}$ der nach der intravenösen Verbenininjektion sezernierten Milch angefeuchtet und nach Trocknung in einem Soxhletschen Apparat mit Äther lange Zeit extrahiert. Die erhaltene Lösung wurde von Äther befreit, der Rückstand in Wasser aufgenommen und die wässerige Lösung konzentriert. Die konzentrierte Lösung hatte aber keinen bitteren Geschmack und nicht die Fähigkeit, ammoniakalische Silbernitratlösung in der Wärme zu reduzieren. Das Glykosid ist also in der Milch, die nach seiner Darreichung sezerniert wird, nicht enthalten, was darauf beruhen muss, dass das Glykosid entweder im Tierkörper zersetzt wird oder von den Milchdrüsen nicht ausgeschieden werden kann. Um diese Frage entscheiden zu können, wurde ein Versuch am Harn eines Kaninchens, dem $50 \mathrm{mg}$ Verbenin pro $\mathrm{kg}$ Gewicht intravenös gegeben worden waren, in Bezug auf das Glykosid angestellt. Der in 24 Stunden nach der Darreichung gelassene Harn wurde nach Neutralisierung mit Salzsäure eingedampft und der Rückstand in absolutem Alkohol gelöst. Die alkoholische Lösung wurde von einem Stück Filterpapier aufgesaugt, das nach Trocknung in der oben gesagten Weise mit Äther extrahiert wurde. Die Ätherlösung wurde vom Lösungsmittel befreit und der Rückstand in $1 \mathrm{ccm}$ Wasser gelöst. Die wässerige Lösung schmeckte nicht bitter und reduzierte ammoniakalische Silbernitratlösung beim Erwärmen kaum. Deshalb ist es sicher, dass das einverleibte Verbenin als solches kaum im Harn abgesondert wird. Deshalb muss man annehmen, dass das Glykosid im Körper zersetzt wird. Dass die galaktogoge Wirkung des Glykosides aber lang anhaltend ist, scheint darauf hinzudeuten, dass das dargereichte Glykoside in gewissen Geweben fixiert und dort allmählich zersetzt wird.

\section{Zusammenfassung.}

Verbena officinalis L. enthält neben einem schon dargestellten Glykosid, dem Verbenalin, noch ein neues, das nicht in allen, doch in vielen chemischen sowie pharmakologischen Eigenschaften dem ersteren sehr nahe steht, und dem infolgedessen ein neuer Namen ,Ver- 
benin" gegeben wird. Das Verbenin wirkt bei Fröschen auf die sympathischen Nervenenden der Hautschleimdrüsen, des Herzens und der Gefässe in kleinen Mengen erregend, aber in grossen lähmend. In recht grossen Dosen lähmt es das Zentralnervensystem. Bei Kaninchen wirkt es ebenfalls auf die fördernden sympathischen Nervenenden des Uterus und der Speicheldrüsen in dieselben Weise. Bei melkenden Tieren bewirkt es in Dosen, die eine nachweisbare Veränderung der Allgemeinerscheinungen nicht hervorzurufen imstande sind, eine starke und zwar lang anhaltende Vermehrung der Milchsekretion, was davon überzeugt, dass die Droge als ein Galaktagogum wirksam sein kann, wie man früher geglaubt hat. Das Glykosid scheint nach dem Eintreten ins Blut in gewissen Geweben fixiert und dort allmählich zersetzt zu werden. 